\title{
Treatment Effects upon Prolactin and Soluble Receptor of Interleukin-2 in Psoriatic Patients
}

Delia BOTEZATUa, Mihaela TOVARUa, b, Simona-Roxana GEORGESCU ${ }^{\mathrm{a}, \mathrm{b}}$, Antoanela CURICI ${ }^{\mathrm{c}}$, Calin GIURCANEANU ${ }^{\mathrm{b}, \mathrm{d}}$

aDepartment of Dermato-Venereology, "Dr. Victor Babes" Clinical Hospital of Infectious and Tropical Diseases, Bucharest, Romania

"Carol Davila" University of Medicine and Pharmacy, Division of Dermato-Venereology, Bucharest, Romania

'Synevo, Central Laboratory of Bucharest, Romania

dELIAS University Emergency Hospital, Department of Dermato-Venereology, Bucharest, Romania

ABSTRACT
Background: Psoriasis vulgaris is a chronic inflammatory hyper-proliferative disease of the skin, scalp,
nails, and joints. It has been hypothesized that prolactin (PRL) may modulate the skin immune system and
be involved in the pathogenesis of psoriasis. Psoriasis exerts significant, negative impact on patients' quality
of life. Relatively high rates of depression are reported in patients with psoriasis.
Objectives: The aim of this work was to study the possible role of PRL in the pathogenesis of psoriasis and
its correlation with the disease activity, clinically, molecular and emotional status of patients.
Subjects and methods: A total of 41 samples were analyzed - 21 new patients with psoriasis vulgaris
before treatment and 20 after therapy - were included in this study. In all patients, we determined skin
disease activity according to the PASI index, the psychological impact measured with HAMA and HAMD
scales and the quality of their life measured by DLQI. The concentration of prolactin in the serum was
measured by enzyme-linked immunosorbent assay (ELISA), and the concentration of soluble receptor of
IL-2 was measured with automated immune chemiluminescent system - IMMULITE procedure.
Results: The PRL and sIL2R serum levels were significantly decreased after three months of therapy, at
least $50 \%$ with a p value $<0.00001$. Clinical, hormonal, molecular correlations between before and after therapy
were measured with a statistically significant result. Correlations between HAMA-PRL and DLQI-PRL before
therapy were not statistically significant, only the relationship between HAMD and PRL was demonstrated.
After treatment, we obtained a significant clinical, psychological and paraclinical (especially serum levels of
prolactin and sIL2R) decreased and relevant response on all the patients treated and analyzed.

Address for correspondence:

Delia Botezatu

"Dr. Victor Babes" Clinical Hospital,

Mihai Bravu Str, No 281, Postal Code 030303, Bucharest, Romania

Tel.: 0040762319456

E-mail:delia_botezatu@ymail.com

Article received on the $19^{\text {th }}$ of January 2018 and accepted for publication on the $26^{\text {th }}$ of March 2018. 
Conclusion: Prolactin seems to have a role in the pathogenesis of psoriasis and may represent a cause and/or a consequence of psoriasis pathology. The most likely scenario is that PRL enhances interferon-induced chemokine production in keratinocytes, thereby facilitating cutaneous T-cell infiltration. This raises the intriguing light that PRL may offer a novel future therapeutic target in psoriasis and other skin diseases that worsen in response to psychological distress.

Keywords: prolactin, interplay between prolactin and sIL2R, psoriasis vulgaris.

\section{INTRODUCTION}

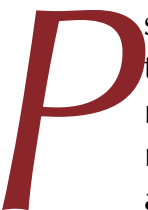
soriasis vulgaris is a T-cell mediated autoimmune disease (1). Genetic, environmental, immune defect and hormonal factors take part in the autoimmune pathogenesis of disease (2). Evidence for the central role of T helper (Th1) lymphocytes comes from both animal models of psoriasis and trials of treatment with T-cell inhibitors (3). There is some evidence that psoriasis can worsen at ages when hormonal changes (e.g., puberty and menopause) occur, and may also improve or worsen during pregnancy (4).

Prolactin (PRL), as a neuropeptide secreted by the anterior pituitary gland, possesses a variety of physiological actions. It has been implicated as an essential immunomodulator and exerts a proliferative effect in cultured human keratinocytes via specific receptors. Studies have indicated an increase in serum PRL levels in psoriasis and an exacerbation of psoriasis when a prolactinoma is present $(5,6)$. More than three decades ago, it was first asserted that bromocriptine, an ergoline derivative and dopamine agonist, induced respite of psoriatic lesions, even psoriatic arthritis (7). This theory enlightened concepts to demonstrate a role of prolactin in the pathogenesis of this chronic inflammatory disease (8). As mentioned, the pathological hallmarks of psoriasis are keratinocyte hyperproliferation, dysregulated angiogenesis and expression of proinflammatory $\mathrm{T}$ helper (Th) 1 cytokines (9). Psoriasis vulgaris is often triggered or exacerbated by psychoemotional stress $(10,11)$. Given that PRL represents a classic neuroendocrine mediator of stress responses, it has been hypothesized that effects of stress on psoriasis are controlled by modifications in serum PRL levels (8). Due to the modest number of reported cases showing a correlation between hyperprolactinemia and psoriasis vulgaris (12-14), this association remains to be proven. Even though increased prolactin serum levels have been reported in patients with psoriasis, frank evidence that the incidence of psoriasis is increased in patients with hyperprolactinemia is lacking (15).

\section{Aim of the work}

The aim of this work was to study the possible role of PRL in the pathogenesis of psoriasis and its correlation with a chosen Th1 cytokine with disease activity and patients' emotional status.

\section{PATIENTS AND METHODS}

This study included 21 new cases with chronic generalized psoriasis, 11 males and 10 females aged between 13 and 75, with a mean of $51.23 \pm 3.41$ (44.10 to 58.37), followed from November 2016 to June 2017. Patients have provided their written informed consent (preand post-therapy). The study and its consent procedure were approved by the relevant Ethics Committee of the hospital. Approval of the study was also specifically obtained from the ethics committees of "Carol Davila" University of Medicine and Pharmacy and "Dr. Victor Babeş" Hospital of Infectious and Tropical Diseases, Bucharest. Diagnostic of psoriasis vulgaris was made based on clinical and histopathological exams. The process of recruiting patients with psoriasis vulgaris is summarized in Figure 1.

Patients were subjected to:

1- Full history taking: Including personal history, history of the disease (age at onset, duration and extension of the disease), past history (history of drug intake, lactation, menstrual irregularities in females and andrological complaints in males) and family history.

2- Full clinical examination: A complete dermatological examination was done for each patient in order to determine the extent and distribution of the disease. Clinical severity of psoriasis was assessed by using the Psoriasis Area Severity Index (PASI) score. Points for 
erythema, infiltration and desquamation of the skin ranged from 1 to 4 , and the involved area from 1 to 6 ; thus, theoretically, the PASI ranges from 0 to 72 , with higher scores indicating more severe condition. In our study, mild psoriasis was classified as a PASI less than 7, moderate psoriasis as a PASI between 7 and 15 , and severe psoriasis as a PASI of $>15$ (16). The following patients were excluded from the study: hepatic and renal patients, pregnant or lactating females, patients who were receiving any medications affecting PRL such as phenothiazine (chlorpromazine), antidopaminergic agents (metoclopramide), antihypertensive agents (calcium channel blockers, methyldopa) and $\mathrm{H} 2$ blockers (cimetidine) and endocrinopathies, those with no topical or systemic treatment prior to enrollment (a period of four weeks for topic therapy and three months for systemic medication).

3- Therapeutic management: Each patient received therapy: 17 of them were given combined therapy [topical and systemic with $15 \mathrm{mg} /$ weekly dose of Methotrexate (MTX)], three of them only systemic treatment (they refused ointment use) and one patient only topical therapy; all subjects were compliant to medication for 12 weeks.

4- Clinical assessment: It consisted in determination of the clinical response after 12 weeks (three months), which was evaluated by using PASI score.

5- Specific and generic measures of psychological distress: Two psychological instruments were used. Firstly, the Hamilton Depression Scale (HDS, HAMD or HAD), which is a depression test measuring the severity of clinical depression symptoms; it consists of 21 items, each defined by series of symptoms; some items were rated on a 5-points scale, ranging from 0 (not present) to 4 (severe), while others were rated on a 3 -points scale, ranging from 0 (not present) to 2 (severe); scoring is based on the first $17(0-7=$ Normal, $8-13=$ Mild depression, $14-18=$ Moderate depression, 19-22=Severe depression, $\geq 23=$ Very severe depression) (17). Secondly, we used the Hamilton Anxiety Scale (HAMA), a rating scale developed to quantify the severity of anxiety symptomatology; it consists of 14 items, each defined by a series of symptoms; each item is rated on a 5-point scale, ranging from 0 (not present) to 4 (severe), with a total score range of $0-56$, where $<17$ indicates mild severity, 18-24 mild to moderate severity, and 25-30 moderate to severe (18).

We screened for psoriatic arthritis (PsA) to exclude subjects with any current or past musculoskeletal manifestations. The diagnosis of PsA was made by experienced rheumatologists and evaluated by DAPSA (disease activity in psoriatic arthritis) score (19).

Furthermore, patients were also asked to answer a DLQI (dermatology life quality index) (20) questionnaire before and after therapy, just like for HAMA and HAMD scales.

6- Laboratory investigations: all patients were subjected to the following investigations: complete blood picture, liver and renal function tests.

As the PRL serum levels reflect physiological diurnal variation, the blood samples were taken in the morning hours (at least one hour after waking up) and after at least 20 minutes at rest before sampling. We used the immunoassay for in vitro quantitative determination of PRL in human serum, and also "ECLIA" electrochemiluminescence immunoassay on Elecsys and cobase immunoassay analyzers, which is a "sandwich method" with a total duration of 18 minutes. For the latter technique, samples $(10 \mu \mathrm{L})$ were incubated with $1^{\text {st }}$ biotinylated monoclonal prolactin-specific antibody to form a first complex. After addition of a monoclonal prolactin-specific antibody labeled with a ruthenium complex and streptavidin-coated microparticles, a sandwich complex is formed and becomes bound to the solid phase via interaction of biotin and streptavidin. The reaction mixture is aspirated into the measuring cell, where the microparticules are magnetically captured onto the surface of the electrode. Unbound substances are removed with ProCell/ProCellM. Application of a voltage to the electrode then induces chemiluminescent emission, which is measured by a photomultiplier. Results were determined via a calibration curve, which is instrument-specifically generated by 2-point calibration and a muster curve provided via the reagent barcode. The limit of sensitivity of the assay was $1.00 \mu \mathrm{lU} / \mathrm{mL}(0.047 \mathrm{ng} / \mathrm{mL})$. In the test there was no cross reactivity to other human hormones (hLH, hFSH, hTSH, hCG and HPL).

The receptor of the cytokine interleukin 2 (IL-2) (sIL2R) plays a crucial role in the regulation 
of immune response. Binding of IL-2 to its receptor (IL2-R) on the surface of T-lymphocytes triggers a series of intracellular signaling events that result in the activation and proliferation of resting $\mathrm{T}$ cells and, ultimately, in the generation of helper, suppressor and cytotoxic T cells, which mediate immune reactions. The IL-2 receptor is unique among growth factor receptors in that it is made up of at least three distinct membrane components: an $\alpha$-chain (IL2R $\alpha)$, the so-called Tac antigen (55 kDa), a $\beta$-chain (IL2R $\beta, 70-75 \mathrm{kDa}$ ) and a $\gamma$-chain (IL2R $\gamma, 64 \mathrm{kDa}$ ). Different combinations of these three components generate various forms of the IL-2 receptor, each of them manifesting different binding affinities to IL-2. Most resting T cells, B cells, large granular lymphocytes (LGLs) and monocytes do not express significant numbers of this receptor on their surfaces. Upon activation, receptor molecules are expressed on the surface of the cells, and a soluble form (sIL2R) is released, which is about $10 \mathrm{kDa}$ smaller than the membrane-bound protein. It has been found that SIL2R was present at low levels in the serum of healthy individuals and at significantly elevated levels in a broad range of disorders, involving immune activation. We provide here a short description of the procedure. IMMULITE/IMMULITE 1000IL2R is a solid-phase, two-site chemiluminescent immunometric assay. Incubation cycles $-1 \times 30$ minutes. It was used an ultracentrifuge to clear lipemic samples. The sample cups contained at least $100 \mu \mathrm{L}$ of serum, more than the volume required of $50 \mu \mathrm{L}$ of serum. This was according to the manufacturer's instructions (Siemens, Germany).

\section{STATISTICS}

The he data were collected and introduced to OpenOffice PC, version 4.1.1 Copyright (C) 2014 The Apache Software Foundation. The statistical analysis was shaped with the following programs: - R version 3.4.0 (2017-04-21) -- "You Stupid Darkness" Copyright (C) 2017 The R Foundation for Statistical Computing Platform R Core Team (2017). R: A language and environment for statistical computing. R Foundation for Statistical Computing, Vienna, Austria. URL https://www.R-project.org/., Inc. Besides standard R, we also used A Collection of Statistical Tools for Biologists. R package version 1.3-1.https://CRAN.R-project.org/package $=$ asbio, asympTest: a simple $\mathrm{R}$
Patients with psoriasis assessed for eligibility $(n=30)$

Excluded $(n=9)$ :

Not meeting the inclusion criteria $(n=8)$

Declined participation $(\mathrm{n}=1)$

Completed the study and analysis $(n=20)$

Total number of patients pre- + post-therapy $=41$

FIGURE 1. The process of recruiting patients with psoriasis vulgaris

package for performing classical parametric statistical tests and confidence intervals in large samples The $\mathrm{R}$ Journal (Software: R Package, asympTest, version 0.1.2). si ggplot2 (c) H. Wickham. ggplot2: Elegant Graphics for Data Analysis. Springer-Verlag New York, 2009. Python version 3.6.0 with graphics and scientific modules on Anaconda(c) 2016 Continuum Analytics distribution was helpful too.

Inferential statistics. Given that each of the followed parameters in both groups represents two random and independent variables, the combo algorithm between them was as follows: for binomial variables, if each possible value of the random variables was at least five times in the study, it was considered that the binomial distribution of the variable could be approximated by a Gaussian distribution. For statistical inferential, a bidirectional $\chi^{2}$ (chi square) test was used to test two independent proportions; if the distribution of the binomial variable could not be approximated with a normal one for comparison, a Fisher test was exactly bidirectional for independent proportions. If the distribution of the binomial variable could not be approximated with a normal one for comparison, a Fisher test was exactly bidirectional for independent proportions. For continuous variables, if the distributions of the variables on both batches could be approximated with the normal distribution, a Welch T bidirectional test was used for the difference of the averages for two independent normal distributions. If the distributions could not be approximated with the normal distribution, a Wilcoxon Rank-Sum test for the difference of localization 
between two independent distributions was utilized. For the comparison of pre- and post-therapy parameters in patients with psoriasis analysis, we used tests for correlated samples, namely: if distributions were close to Gaussian distribution, a Paired T test was used, while for distributions distant from normal distribution a Wilcoxon Signed Rank was needed.

The levels of prolactin and soluble receptor of IL-2 were normally distributed, and therefore, the student's test was used to analyse the differences between the two groups. Spearman correlation coefficient was used for correlation between PRL and selected variables. Data are presented as the mean \pm standard deviation. A $p$ value less than 0.05 was considered to be statistically significant. Chi square test was used for the variables of demographic data.

\section{RESULTS}

\section{Clinical results}

n this study, the duration of psoriasis vulgaris ranged from one year to 21 years, with a mean of $8.04 \pm 1.29$ years. With regard to family history, two out of the 21 patients had a positive family history (9.52\%). According to PASI score, 20 (95.23\%) patients were classified as severe psoriasis; after therapy, 13 of them became moderate and six mild, one patient (4.76\%) was classified as moderate psoriasis and became mild; one patient in the group with severe disease did not come for post-therapy evaluation. There was a significant decrease in the values of PASI score before and after therapy (Table 1).

\section{Laboratory results}

In the group of patients, serum levels of PRL ranged from $184 \mu \mathrm{UI} / \mathrm{mL}$ to $636 \mu \mathrm{UI} / \mathrm{mL}$

\begin{tabular}{|l|l|l|l|l|l|}
\multicolumn{2}{c|}{$\begin{array}{c}\text { PASI score } \\
\text { before } \\
\text { treatment }\end{array}$} & \multicolumn{4}{|c|}{ PASI score after treatment } \\
\cline { 2 - 6 } Moderate & Mild & Moderate & Severe & Total \\
\cline { 2 - 6 } & $\%$ & 1 & 0 & 0 & 1 \\
\hline \multirow{2}{*}{ Severe } & N & 6 & 13 & 1 & 20 \\
\hline \multirow{2}{*}{ Total } & $\%$ & 30 & 65 & 5 & 95.23 \\
\hline & N & 7 & 13 & 0 & 20 \\
\hline & $\%$ & 35 & 65 & 0 & 100 \\
\hline
\end{tabular}

TABLE 1. The values of PASI score before and after treatment

\begin{tabular}{|c|c|c|c|}
\hline & \multicolumn{2}{|c|}{ Prolactin } & \multirow{2}{*}{$\begin{array}{l}\text { Test } \\
\text { Shapiro-Wilk } \\
\text { p value }\end{array}$} \\
\hline & Range & Mean \pm SD & \\
\hline $\begin{array}{l}\text { Before } \\
\text { treatment }\end{array}$ & $184-636$ & $362.55 \pm 27.85$ & 0.812 \\
\hline $\begin{array}{l}\text { After } \\
\text { treatment }\end{array}$ & $100-305$ & $133 \pm 10.57$ & 0.0001 \\
\hline
\end{tabular}

TA B LE 2. Comparison between serum PRL levels before and after therapy

\begin{tabular}{|l|l|l|l|}
$\begin{array}{c}\text { Median } \\
\text { PRL } \\
\text { pre-therapy }\end{array}$ & $\begin{array}{c}\text { Median PRL } \\
\text { post-therapy }\end{array}$ & p value & $\begin{array}{l}\text { Median of } \\
\text { differences } \\
\text { (95\% CI) }\end{array}$ \\
\hline 370.50 & 119.50 & $<0.00001$ & $\begin{array}{l}250.00 \\
(202.50 \text { to } \\
297.50)\end{array}$ \\
\hline
\end{tabular}

TA B LE 3. P value of serum levels of PRL pre-/post therapy

$(452.00 \mu \mathrm{UI} / \mathrm{mL})$, with a mean and SD of 362.55 $\pm 27.85 \mu \mathrm{UI} / \mathrm{mL}$ (324.23 to 440.86 ). The serum levels of PRL after therapy ranged from $100 \mu \mathrm{UI} / \mathrm{mL}$ to $305 \mu \mathrm{UI} / \mathrm{mL}(205.00 \mu \mathrm{UI} / \mathrm{mL})$, with a mean and SD of $133 \pm 10.57 \mu \mathrm{UI} / \mathrm{mL}$ (110.87 to 155.12), which meant a significant decrease in comparison with the values measured before treatment: $p<0.00001$ (Tables 2 and 3).

In the group of patient, pre-therapy serum levels of sIL2R ranged from $311 \mathrm{U} / \mathrm{mL}$ to $2766 \mathrm{U} / \mathrm{mL}$, with a mean and SD of $710.05 \pm 117.88 \mathrm{U} / \mathrm{mL}$ (463.31 to 956.78). After 12 weeks of therapy, serum levels of sIL2R ranged from $100 \mathrm{U} / \mathrm{mL}$ to $751 \mathrm{U} / \mathrm{mL}$, with a mean and SD of $268.60 \pm 33.98 \mathrm{U} / \mathrm{mL}$ (197.46 to 339.73), showing also an important decline comparatively to pre-therapy levels: $p<0.00001$ (Tables 4 and 5).

\begin{tabular}{|c|c|c|c|}
\hline & \multicolumn{2}{|c|}{ sIL2R } & \multirow{2}{*}{$\begin{array}{l}\text { Test } \\
\text { Shapiro-Wilk } \\
\text { p value }\end{array}$} \\
\hline & Range & Mean $\pm S D$ & \\
\hline $\begin{array}{l}\text { Before } \\
\text { treatment }\end{array}$ & $311-2766$ & $710.05 \pm 117.88$ & 0.0001 \\
\hline $\begin{array}{l}\text { After } \\
\text { treatment }\end{array}$ & $100-751$ & $268.60 \pm 33.98$ & 0.0001 \\
\hline
\end{tabular}

TA B LE 4. Comparison between serum sIL2R levels before and after therapy

\begin{tabular}{|c|c|l|l|}
$\begin{array}{c}\text { Median } \\
\text { sIL2R } \\
\text { pre-therapy }\end{array}$ & $\begin{array}{c}\text { Median } \\
\text { sIL2R } \\
\text { post-therapy }\end{array}$ & p value & $\begin{array}{l}\text { Median of } \\
\text { differences } \\
(\mathbf{9 5 \%} \text { CI) }\end{array}$ \\
\hline 560.00 & 227.00 & $<0.00001$ & $\begin{array}{l}358.00 \\
(296.50 \text { to } \\
451.00)\end{array}$ \\
\hline
\end{tabular}

TABLE 5. Relevance of semnificative $p$ value of SIL2R 


\section{Psychological results}

The values of HAMA score before therapy ranged from 6.0 to 25.0, with a mean and SD of $16.50 \pm 1.15$, while after therapy they significantly decreased, ranging from 3.0 to 11.0 , with a mean and SD of 7.90 $\pm 0.46 \quad(p<0.00001)$ (Table 5). The values of HAMD score before therapy ranged from 9.0 to 23.0, with a mean and SD of $15.80 \pm 0.91$, while after therapy they significantly decreased, ranging from 3.0 to 11.0 , with a mean and SD of $6.45 \pm 0.48(\mathrm{p}<0.00001)$ as shown in (Table 5). The values of DLQI score before treatment ranged from 7.0 to 28.0, with a mean and SD of $17.60 \pm 1.35$, while after treatment, they showed an important decline, ranging from 1.0 to 12.0, with a mean and SD of $6.95 \pm 0.75(p<0.00001)$, as shown in Table 6 .

Correlations between serum levels of prolactin and soluble receptor of IL-2 before and after therapy were statistically significant, as detailed in Table 6. For this purpose, the following linear correlation indices were calculated: $r$ Pearson if the distribution of the variables could be approximated with the normal distribution, or $p$ Spearman if the distributions were offset by Gaussian distribution. The results were considered statisti-

\begin{tabular}{|c|c|c|c|c|}
\hline \multirow[b]{3}{*}{$\begin{array}{l}\text { Before } \\
\text { treatment }\end{array}$} & \multicolumn{2}{|c|}{ HAMA } & \multirow{2}{*}{\multicolumn{2}{|c|}{$\begin{array}{l}\text { Test } \\
\text { Shapiro-Wilk } \\
\text { (P value) } \\
\text { Paired T-test }\end{array}$}} \\
\hline & \multirow{2}{*}{$\begin{array}{l}\text { Range } \\
6.0-25.0\end{array}$} & \multirow{2}{*}{$\begin{array}{l}\text { Mean } \pm \text { SD } \\
16.50 \pm 1.15\end{array}$} & & \\
\hline & & & 0.677 & \\
\hline \multirow[t]{3}{*}{$\begin{array}{l}\text { After } \\
\text { treatment }\end{array}$} & $3.0-11.0$ & $7.90 \pm 0.46$ & 0.08 & 0.00001 \\
\hline & \multicolumn{2}{|c|}{ HAMD } & \multicolumn{2}{|c|}{ Paired T-test } \\
\hline & Range & Mean \pm SD & & $\mathrm{P}$ value \\
\hline $\begin{array}{l}\text { Before } \\
\text { treatment }\end{array}$ & $9.0-25.0$ & $15.80 \pm 0.91$ & 0.223 & \\
\hline \multirow[t]{3}{*}{$\begin{array}{l}\text { After } \\
\text { treatment }\end{array}$} & $3.0-11.0$ & $6.45 \pm 0.48$ & 0.681 & 0.00001 \\
\hline & \multicolumn{2}{|c|}{ DLQI } & \multicolumn{2}{|c|}{ Paired T-test } \\
\hline & Range & Mean \pm SD & & P value \\
\hline $\begin{array}{l}\text { Before } \\
\text { treatment }\end{array}$ & $7.0-28.0$ & $17.60 \pm 1.35$ & 0.575 & \\
\hline $\begin{array}{l}\text { After } \\
\text { treatment }\end{array}$ & $1.0-12.0$ & $6.95 \pm 0.75$ & 0.191 & 0.00001 \\
\hline
\end{tabular}

TA B LE 6. Comparison between values of HAMA, HAMD and DLQI cally significant at a sensitivity level of $\alpha=0.05$, which means $p<0.05$ (shown in Table 7).

Correlations between HAMA-PRL and DLQI-PRL before therapy were not statistically significant - only therelationship between HAMD and PRL was demonstrated (Table 8). After treatment, we obtained a significant clinical, psychological and paraclinical (serum levels of prolactin and sIL2R specially) decreased and relevant response on all the patients treated and analyzed.

We found representative relationship between clinical and psychological response, PASI correlated with HAMA and HAMD scales. Correlations between PASI-DLQI were also statistically significant (Table 9).

We also analyzed the data after therapy, and therefore the results are redundant; they were also statistically significant, but quite logical, considering that all patients responded to therapy and the lab exams with clinical and psychological responses were decreased by at least $50 \%$.

\begin{tabular}{|l|l|l|}
\hline $\begin{array}{l}\text { Correlations between } \\
\text { PRL and sIL2R }\end{array}$ & p Spearman & p value \\
\hline Before treatment & 0.775 & $<0.0001$ \\
\hline After treatment & 0.712 & $<0.0001$ \\
\hline
\end{tabular}

TA B LE 7. Relationship between PRL and sIL2R before and after treatment

\begin{tabular}{l|l|l|}
\multicolumn{2}{|l|}{ Correlations Pre-therapy } & Prolactin \\
\hline HAMD & r Pearson & 0.520 \\
& p value & 0.01 \\
\hline \multirow{2}{*}{ HAMA } & r Pearson & 0.210 \\
\hline \multirow{2}{*}{ DLQI } & p value & 0.372 \\
\hline & r Pearson & 0.161 \\
\hline & p value & 0.496 \\
\hline
\end{tabular}

TA B L E 8. Correlations between HAMA, HAMD, DLQI and prolactin before therapy

\begin{tabular}{|l|l|l|}
\hline Correlations Pre-therapy & PASI \\
\hline \multirow{2}{*}{ HAMD } & p Pearson & 0.497 \\
& p value & 0.025 \\
\hline \multirow{2}{*}{ HAMA } & p Pearson & 0.721 \\
\hline \multirow{2}{*}{ DLQI } & p value & 0.0001 \\
\hline & p Pearson & 0.849 \\
\hline
\end{tabular}

TA B LE 9. Significant clinical and psychological relationship pre-therapy 


\section{DISSCUSION}

Doriasis vulgaris is often triggered or exacerbated by psychoemotional stress $(10,11)$. It has been hypothesized that effects of stress on psoriasis are controlled by modifications in serum PRL levels (8). Dunna and Finlay observed that, during pregnancy, female patients with psoriasis tended to be stable and on the early months postpartum, with a potential worsening of their psoriatic lesions, which led to the presumption that there may be a link between physiological hyperprolactinemia associated with lactation and the exacerbated course of psoriatic lesions (21).

So, the aim of this study was to study the possible role of PRL in the pathogenesis of psoriasis and its correlation with disease activity, serum levels of a Th1 cytokine receptor and patients' emotional status.

Our results revealed that PRL serum levels were significantly higher in psoriatic patients before conventional therapy, being significantly reduced after treatment; also, they showed a correlation between serum PRL levels and PASI. Furthermore, we found important pre-treatment higher serum values of Th1 cytokine receptor, a soluble receptor of interleukin-2 (sIL2R), with a relevant decline after therapy and correlation with PASI score. Also, correlations between PRL and SIL2R before and after therapy were statistically significant, with a $p$ value $<0.0001$, which can suggest an important and possible role of prolactin in stimulation of Th1 cytokines in psoriasis vulgaris.

Few studies about sIL2R as a marker of disease severity in psoriasis vulgaris were found in the literature. De Rie, et al. found that sIL2R levels were well correlated with disease activity in patients with PV and closely followed the decrease of disease activity during immunosuppressive therapy (22). Duncan et al. revealed that serum levels of sIL2R decreased after treatment with PUVA (psoralen-UVA) and Cyclosporine therapy, but did not change with NB-UVB (narrow-band UVB) treatment $(23,24)$.

Our results are consistent with those of DilmeCarreras et al. (12), whose study included 20 patients with psoriasis and 20 healthy controls. Prolactin levels were measured by Enzyme-linked Immuno Assay (EIA) in the serum of patients with psoriasis before and after treatment with Tacalcitol ointment $4 \mu / g$ once a day for six weeks, and disease severity was assessed by PASI in all patients. Serum levels of PRL were significantly higher in patients than controls before treatment. The authors also found that PRL levels were significantly reduced after treatment with Tacalcitol and there was a correlation between pretreatment serum PRL levels and PASI. El-Khateeb, et al., who initiated a study including 15 psoriasis patients and 15 healthy volunteers as controls, the PASI score was evaluated, and PRL levels in serum and blister fluid were assessed by (ELISA). PRL levels were significantly elevated in blister fluid of psoriatic lesional skin. Correlations between PASI score and different serum PRL levels in lesional and non-lesional skin were insignificant. Significant positive correlations of PRL level were observed between lesional and non-lesional skin in psoriasis and between serum and clinically normal skin in both psoriasis and control subjects (25).

In 2000, Regana and Millet reported three cases of women with plaque-type psoriasis whose severity and extent of skin lesions correlated with the development of a prolactinoma (14). All three patients were treated with bromocriptin. They had normalization of PRL level and also improvement of psoriatic lesions. Then, they discontinued bromocriptin and all cases had relapsed in psoriasis. In 2015, Husakova et al. demonstrated a correlation between increased PRL serum levels and psoriatic arthritis; therefore, elevated PRL serum levels might represent a marker of inflammatory joint disease in patients suffering from psoriasis vulgaris (26).

Our results revealed also that values of HAMA and HAMD score were significantly high in all psoriatic patients and significantly decreased after treatment, and there was a strong correlation between HAMD and PASI. These results are in accordance with those reported by Verhoeven, et al., who found a significant association between stress and disease severity. This prospective study of 41 psoriasis patients showed that high levels of daily stressors were related to an increase in disease severity 12 weeks later (27). Unlike the above mentioned studies, Zachariae et al. showed that stress responders significantly differed from non-stress responders. Stress responders tended to self-report greater disease severity than non-stress responders, even though clinical measures of disease severity (e.g., PASI) did not vary between groups. However, stress re- 
sponders were found to have more plaques of psoriasis on visible areas than non-visible regions (28).

The limitations of our study include the relatively small number of subjects analyzed and the lack of immunohistochemistry for PRL receptors on skin biopsies in PV patients. Local PRL values might reflect disease activity better than systemic ones.

Girolomoni et al. showed that PRL stimulates human epidermal keratinocytes proliferation (6), as well as VEGF in vitro production, as demonstrated by Garcia de la Torre et al. (29), and PRL exerts varied proinflammatory activities (30). Furthermore, PRL may contribute to the development of psoriatic plaques by stimulating interferon $\gamma(\mathrm{IFN}-\gamma)(31)$, but in the same time, the inhibition of T-supressor cell functions by PRL may also serve to facilitate the advancement of psoriatic lesions $(9,32)$. As mentioned, PRL incites the production of certain chemokines in human keratinocytes, potentially facilitating Th1 cell infiltration into epidermis and supporting the development of psoriatic plaques $(8,33,34)$. Moreover, Kanda and Watanabe observed that, by activating STAT1, NF- $\kappa$ B and IRF-1, PRL enhanced IFN- $\gamma$-induced transcription and secretion of key chemokines (CXCL9, CXCL10, CXCL11), hence promoting the infiltration of type 1-T helper cells into psoriatic lesions (33). Due to that, cyclosporine $\mathrm{A}(\mathrm{Cs} \mathrm{A})$ is an efficient treatment for psoriasis (35). According to Hiestand et al., PRL competes with CsA for a common binding site on T lymphocytes and prolactin secretion also inverses the immunosupression induced by CsA (36). Neidhart et al. demonstrated that CsA inhibited PRLmediated induction of ornithine decarboxylase and also that CsA and bromocriptine had synergistic effects on autoimmune diseases (37). Therefore, this idea might allow lower-dose CsA thera- py in psoriasis (32). It is possible that differences in peripheral or lymphocyte, rather than pituitary production of PRL, may worsen disease activity in certain patients. Of the currently available prolactin-lowering drugs, bromocriptine at least has been shown to decrease both peripheral and pituitary PRL production and may represent a useful adjunctive therapy in certain patients, particularly in those with refractory disease (38). Recently, it has been shown that PRL enhanced inflammation and Th1 and Th17 cytokine production in a mouse model with imiquimod-induced psoriasiform skin changes (39). PRL is overexpressed in psoriatic skin lesions (25).

In further PRL human studies on PV, it will probably be necessary to divide patients according to gender and concomitant treatment, evaluate their quality of life, and analyse cutaneous PRL.

Due to the modest number of reported cases with correlation between hyperprolactinemia and psoriasis vulgaris (12-14), this association remains to be proven. Even though increased PRL serum levels have been reported in patients with psoriasis, frank evidence supporting an increased incidence of psoriasis in patients with hyperprolactinemia is lacking.

\section{CONCLUSION}

Prolactin seems to have a role in the pathogenesis of psoriasis. This role may represent a cause and/or a consequence of psoriasis pathology. This raises the intriguing prospect that PRL may offer a novel future therapeutic target in psoriasis and other skin diseases that worsen in response to psychological distress.

Conflicts of interest: none declared.

\section{R EFerences}

1. Ozawa M, Aiba S. Immunopathogenesis of psoriasis. Curr Drug Targets Inflamm Allergy 2004;3:137-144.

2. Langan EA, Griffiths CE, Paus R. Exploring the role of prolactin in psoriasis. Arch Dermatol Res 2012;304:115-118.

3. Gottlieb AB. Novel Immunotherapies for Psoriasis: Clinical Research Delivers New
Hope for Patients and Scientific Advances. Journal of Investigative Dermatology Symposium Proceedings 2004;9:79-83.

4. Mowad CM, Margolis DJ, Halpern AC, Suri B, Synnestvedt M, Guzzo CA. Hormonal influences on women with psoriasis. Cutis 1998;61:257-260.

5. Buskila D, Sukenik $S$, Shoenfeld Y. The
Possible Role of Prolactin In Autoimmunity. American Journal of Reproductive Immunology 1991;26:118-123.

6. Girolomoni G, Phillips JT, Bergstresser PR. Prolactin stimulates proliferation of cultured human keratinocytes. J Invest Dermatol 1993;101:275-279.

7. Weber G, Klughardt G, Neidhardt M. 
Psoriasis and human growth hormone: aetiology and therapy.

Arch Dermatol Res 1981;270:361-365.

8. Paus R. Does prolactin play a role in skin biology and pathology? Med Hypotheses 1991;36:33-42.

9. Lowes MA, Bowcock AM, Krueger JG. Pathogenesis and therapy of psoriasis. Nature 2007;445:866-873.

10. Fortune DG, Richards HL, Kirby B, et al. Psychological distress impairs clearance of psoriasis in patients treated with photochemotherapy. Arch Dermatol 2003;139:752-756.

11. O'Leary CJ, Creamer D, Higgins E, Weinman J. Perceived stress, stress attributions and psychological distress in psoriasis. J Psychosom Res 2004;57:465-471.

12. Dilme-Carreras $E$, Martin-Ezquerra $G$, Sanchez-Regana M, Umbert-Millet $P$. Serum prolactin levels in psoriasis and correlation with cutaneous disease activity. Clin Exp Dermatol 2011;36:29-32.

13. Rathika S, Rajappa M, Chandrashekar L, Munisamy M, Thappa DM. Effect of treatment on prolactin levels in patients with psoriasis vulgaris. Clin Chim Acta 2014;429:212-214.

14. Sanchez Regana $M$, Umbert Millet $P$. Psoriasis in association with prolactinoma: three cases. Br J Dermatol 2000;143:864-867.

15. Botezatu D, Tovaru M, Georgescu SR, Leahu OD, Giurcaneanu C, Curici A. Interplay Between Prolactin and Pathogenesis of Psoriasis Vulgaris. Maedica J Clin Med 2016;11:232-240.

16. Feldman SR, Krueger GG. Psoriasis assessment tools in clinical trials. Ann Rheum Dis 2005;64 Suppl 2:ii65-8; discussion: ii69-73.

17. Williams JB. A structured interview guide for the Hamilton Depression Rating Scale. Arch Gen Psychiatry 1988;45:742-747.

18. Vaccarino AL, Evans KR, Sills TL, Kalali AH. Symptoms of anxiety in depression: assessment of item performance of the Hamilton Anxiety Rating Scale in patients with depression. Depress Anxiety 2008;25:1006-1013.

19. Smolen JS, Schoels M, Aletaha D. Disease activity and response assessment in psoriatic arthritis using the Disease Activity index for PSoriatic Arthritis (DAPSA). A brief review.

Clin Exp Rheumatol 2015;33:S48-S50.

20. Atwan A, Piguet V, Finlay AY, Francis NA, Ingram JR. Dermatology Life Quality Index (DLQI) as a psoriasis referral triage tool. Br J Dermatol 2017;177:e136-e137.

21. Dunna SF, Finlay AY. Psoriasis: improvement during and worsening after pregnancy. Br J Dermatol 1989;120:584.

22. De Rie MA, Zonneveld IM, Witkamp L, Van Lier RA, Out TA, Bos JD. Soluble interleukin-2 receptor (sIL-2R) is a marker of disease activity in psoriasis: a comparison of sIL-2R, sCD27, sCD4, sCD8 and sICAM-1. Acta Derm Venereol 1996;76:357360.

23. de Rie MA, Out TA, Bos JD. Low-dose narrow-band UVB phototherapy combined with topical therapy is effective in psoriasis and does not inhibit systemic T-cell activation. Dermatology 1998;196:412-417.

24. Duncan JI, Horrocks C, Ormerod AD, et al. Soluble IL-2 receptor and CD25 cells in psoriasis: effects of cyclosporin A and PUVA therapy. Clin Exp Immunol 1991;85:293-296.

25. El-Khateeb EA, Zuel-Fakkar NM, Eid SM, Abdul-Wahab SE. Prolactin level is significantly elevated in lesional skin of patients with psoriasis. Int J Dermatol 2011;50:693-696.

26. Husakova M, Lippert J, Stolfa J, et al. Elevated serum prolactin levels as a marker of inflammatory arthritis in psoriasis vulgaris. Biomed Pap Med Fac Univ Palacky Olomouc Czech Repub 2015;159:562-568.

27. Verhoeven EW, Kraaimaat FW, de Jong EM, Schalkwijk J, van de Kerkhof PC, Evers AW. Individual differences in the effect of daily stressors on psoriasis: a prospective study. Br J Dermatol 2009;161:295-299.

28. Zachariae R, Zachariae $H$, Blomqvist $K$, et al. Self-reported stress reactivity and psoriasis-related stress of Nordic psoriasis sufferers. J Eur Acad Dermatol Venereol 2004;18:27-36.

29. de la Torre NG, Turner HE, Wass JA.
Angiogenesis in prolactinomas: regulation and relationship with tumour behaviour. Pituitary 2005;8:17-23.

30. Chikanza IC, Panayi GS. Hypothalamicpituitary mediated modulation of immune function: prolactin as a neuroimmune peptide. Br J Rheumatol 1991;30:203-207.

31. De Bellis A, Bizzarro A, Pivonello R, Lombardi G, Bellastella A. Prolactin and autoimmunity. Pituitary 2005;8:25-30.

32. Foitzik K, Langan EA, Paus R. Prolactin and the skin: a dermatological perspective on an ancient pleiotropic peptide hormone. J Invest Dermatol 2009;129:1071-1087.

33. Kanda N, Watanabe S. Prolactin enhances interferon-gamma-induced production of CXC ligand 9 (CXCL9), CXCL10, and CXCL11 in human keratinocytes. Endocrinology 2007;148:2317-2325.

34. Langan EA, Ramot Y, Goffin V, Griffiths CE, Foitzik K, Paus R. Mind the (gender) gap: does prolactin exert gender and/or site-specific effects on the human hair follicle? J Invest Dermatol 2010;130:886-891.

35. Griffiths CE, Katsambas A, Dijkmans BA, et al. Update on the use of ciclosporin in immune-mediated dermatoses. Br J Dermatol 2006;155 Suppl 2:1-16.

36. Hiestand PC, Mekler P, Nordmann R, Grieder A, Permmongkol C. Prolactin as a modulator of lymphocyte responsiveness provides a possible mechanism of action for cyclosporine. Proc Natl Acad Sci USA 1986;83:2599-2603.

37. Neidhart M. Synergism between longacting bromocryptine microcapsules and cyclosporine $\mathrm{A}$ in the prevention of various autoimmune diseases in rats. Experientia 1996;52:892-899.

38. Chuang E, Molitch ME. Prolactin and autoimmune diseases in humans. Acta Biomed 2007;78 Suppl 1:255-261.

39. Hau CS, Kanda N, Tada Y, Shibata S, Sato S, Watanabe S. Prolactin induces the production of Th17 and Th1 cytokines/ chemokines in murine Imiquimod-induced psoriasiform skin. J Eur Acad Dermatol Venereol 2014;28:1370-1379. 